\title{
Separating and connecting properties of the interface within the renal stem/progenitor cell niche
}

\author{
Will W. Minuth* and Lucia Denk \\ *Correspondence: will.minuth@vkl.uni-regensburg.de

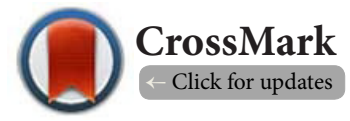

Department of Molecular and Cellular Anatomy, University of Regensburg, Germany.

\begin{abstract}
The renal stem/progenitor cell niche is found during development at the inner side of the organ capsule. A reciprocal exchange of morphogenetic factors between epithelial and mesenchymal cells leads here to a successive anlage of nephrons. It is generally supposed that a close contact exists between involved cells and that transmission of signals occurs via diffusion. However, morphological analysis of specimens fixed in glutaraldehyde (GA) solution reveals that both types of cells are separated by an extended interface. To investigate this special cell arrangement in detail, kidneys of neonatal rabbits were fixed by GA containing lanthanum, alcian blue, cupromeronic blue, ruthenium red or tannic acid. To obtain a comparable view to the niche, parenchyma was cut along the axis of lining collecting ducts for analysis by transmission electron microscopy. Present data illustrate that fixation of specimens by GA including alcian blue, cupromeronic blue, ruthenium red or tannic acid unmasks four different textures of filigree extracellular matrix within the interface. Applied contrasting illuminates a supplementary pattern. Further on, projections of mesenchymal cells lining to epithelial cells are specifically integrated in detected matrix. Experiments are under work to elucidate, whether detected cell to cell contacts via tunneling nanotubes are involved in transmission of morphogenetic signals.
\end{abstract}

Keywords: Kidney, stem/progenitor cell niche, interstitial interface, extracellular matrix, tunneling nanotubes, exchange of morphogenetic factors, transmission electron microscopy, glutaraldehyde, alcian blue, lanthanum, cupromeronic blue, ruthenium red, tannic acid

\section{Introduction}

In the course of an acute or chronic kidney disease important vital functions are lost $[1,2]$. In this life-threatening situation hemodialysis or transplantation of a donor organ are currently available therapeutic options [3,4]. However, the lack of donors and biomedical limitations in dialysis are concrete reasons that the implantation of stem/progenitor cells moves more and more in the focus of research as an innovative form of therapy for the regeneration of diseased renal parenchyma $[5,6]$.

At a first glance it sounds convincing to implant stem/progenitor cells into diseased parenchyma of a kidney $[7,8]$. However, actual data demonstrate that the up to date raised results are meager and intense basic research still has to be performed, until an effective therapy is available [9]. The reasons for that situation are complex. One of the biomedical problems is the only minimal survival of implanted stem/progenitor cells [10]. One has to imagine that in presently performed trials untrained cells are implanted. It is recognized by the fact that before an implantation cells grow in an optimized medium, while after an implantation the situation for them drastically alters. At the site of repair they are exposed to an inflammatory environment including degraded extracellular matrix, toxic metabolites, inadequate nutrition and a low content of oxygen. To overcome this obstacle, possible mechanisms of cell adaptation are presently investigated so that initial seeding of implanted cells within a harmful environment is alleviated [11-16]. In addition, critical analysis of the genuine niche environment informs about specific physiological needs and structural requirements of contained stem/progenitor cells [17].

During development of the kidney the stem/progenitor cell niche is piloting extension of parenchyma [18]. This special cell ensemble is recognized for the first time during anlage of the metanephros. At that early time it consists of the invading ureteric bud and surrounding metanephric mesenchyme [19]. Dichotomous branching of an ureteric bud first leads to the determination of the upper and lower pole and to definition 
Minuth et al. Stem Cell Biology and Research 2014,

of the ventral and dorsal aspect of the organ. Then further branching and in parallel by successive elongation each tip of a bud sets the actual site of nephron formation $[20,21]$. Since the bud represents later the dilated cortical end of a developing collecting duct (CD) tubule, it was suggested to name it CD ampulla [22]. Driven by the mentioned temporal-spatial program and by permanent interactions between epithelial and mesenchymal stem/progenitor cells the parenchyma develops from the inner towards the outer cortex [23]. Until the neonatal period the stem/progenitor cell niche is not distributed at random but keeps an always close contact to the inner side of the organ capsule $[\mathbf{2 4 , 2 5 ]}$.

Within the niche as well epithelial as metanephric mesenchymal stem/progenitor cells are domiciled [26,27]. Epithelial cells are enclosed within the tip of a CD ampulla, while mesenchymal cells surround them as a cap condensate. When the process of dichotomous branching of a CD ampulla approaches its end, intense reciprocal exchange of morphogenetic molecules between both types of stem/progenitor cells takes place leading first to the recruitment of few elected mesenchymal stem/progenitor cells [28]. Then the sorting out initiates formation of the pretubular aggregate developing from a pine-cone to the S-shaped body as first visible signs of proceeding nephron development $[19,29]$.

During the process of nephron induction epithelial cells within the tip of a CD ampulla and surrounding mesenchymal stem/progenitor cells are exactly posted opposite to each other. At this special site morphogenetic molecules such as glial-derived neurotrophic factor (GDNF), hepatocyte growth factor (HGF), epidermal growth factor (EGF) receptor ligands (EGF, HBEGF, TGFa), WNT family members, bone morphogenetic proteins (BMPs), TGF $\beta$, fibroblast growth factors (FGFs) and leukemia inhibitory factor (LIF) are reciprocally transmitted to form the nephron anlage [30-33]. It is generally believed that the exchange of these morphogenetic signals occurs via diffusion. For keeping concentration of diffusing molecules high and to prevent lateral dilution, it is further assumed that epithelial and mesenchymal stem/progenitor cells stay in a close spatial proximity.

However, actual literature shows that both types of stem/ progenitor cells are separated by a striking interstitial interface [34]. Problematic is further that fixation of specimens by conventional glutaraldehyde for transmission electron microscopy does not display any further suspected structural details. Thus, to analyze separation and to unmask hidden features, improved fixation and contrasting of specimens for transmission electron microscopy was applied in the present investigation. The actual data illustrate that projections of mesenchymal cells cross in a special combination with filigree extracellular matrix the interface to contact mesenchymal cells via tunneling nanotubes. Considering traditional diffusion theory and regarding yet detected cell to cell contacts in present results, transmission of morphogenetic factors through the interface are under debate.

\section{Materials and methods Isolation of kidney}

One day old male and female New Zealand rabbits (Seidl, Obern, Germany) were anesthetized with ether and killed by cervical dislocation. Both kidneys including an intact capsule were immediately removed to process them for transmission electron microscopy.

Fixation of specimens for optical and electron microscopy Without delay isolated kidneys were fixed by immersion in glass bottles containing $5 \mathrm{~mL}$ of following solutions:

Series 1: 5\% glutaraldehyde (Serva, Heidelberg, Germany) buffered with $0.15 \mathrm{M}$ sodium cacodylate, $\mathrm{pH}$ 7.4.

Series 2: $5 \%$ glutaraldehyde buffered with $0.15 \mathrm{M}$ sodium cacodylate, $\mathrm{pH} 7.4$.

Series 3: $5 \%$ glutaraldehyde and $1 \%$ alcian blue (Serva) buffered with $0.15 \mathrm{M}$ sodium cacodylate, $\mathrm{pH}$ 7.4.

Series 4: $5 \%$ glutaraldehyde buffered with $0.15 \mathrm{M}$ sodium cacodylate, $\mathrm{pH} 7.4$ followed by incubation with $0.1 \%$ cupromeronic blue (Santa Cruz, Heidelberg Germany) and 0.1 M magnesium chloride hexahy drate (Sigma, Taufkirchen, Germany) dissolved in sodium acetate buffer, $\mathrm{pH}$ 5.6. Counterstaining with $0.5 \%$ sodium tungstate dehydrate (Sigma).

Series 5: $5 \%$ glutaraldehyde and $0.5 \%$ ruthenium red (Fluka, Taufkirchen, Germany) buffered with $0.15 \mathrm{M}$ sodium cacodylate, $\mathrm{pH}$ 7.4.

Series 6: 5\% glutaraldehyde and 1\% tannic acid (Sigma) buffered with $0.15 \mathrm{M}$ sodium cacodylate, $\mathrm{pH}$ 7.4. The period of primary fixation was for 1 day at room temperature. Special fixation and contrasting techniques for transmission electron microscopy was adapted for renal parenchyma from previously published literature $[35,36]$.

\section{Postfixation of specimens}

After several washes with $0.15 \mathrm{M}$ sodium cacodylate the specimens from series 1,3 and 5 were postfixed for $1 \mathrm{~h}$ in the same buffer but containing $1 \%$ osmium tetroxide (Science Services, München, Germany). In series $20.1 \%$ lanthanum III chloride hydrate was still added. In series 6 postfixation was performed with $0.5 \%$ ruthenium red and $1 \%$ osmium tetroxide in $0.15 \mathrm{M}$ sodium cacodylate.

\section{Orientation of parenchyma before embedding}

To obtain a comparable view to both the stem/progenitor cell niche and the neighboring organ capsule, each kidney was first divided between both poles (Figure 1a). When this strategy is followed, the parenchyma is orientated always along the straight cortico-medullary course of lining collecting duct (CD) tubules. Cutting in parallel to this axis an exact vertical view to the renal stem/progenitor cell niche in relation to the covering organ capsule becomes possible (Figure 1b). All of the demonstrated micrographs show this perspective so that comparisons between different experimental series become possible. 
a

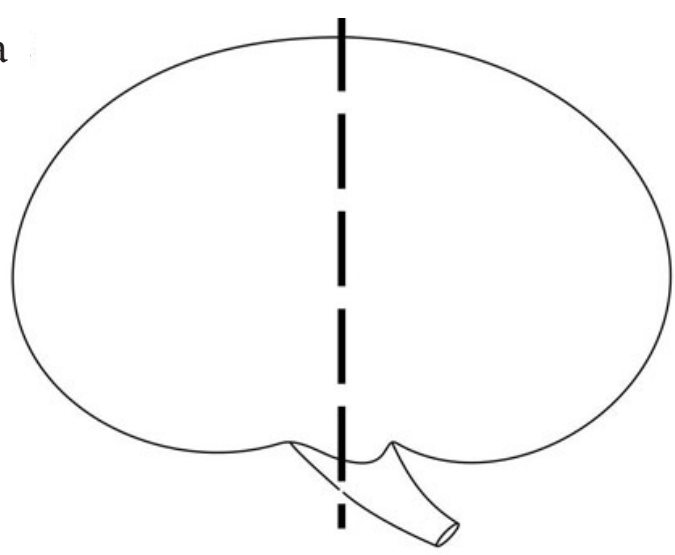

b

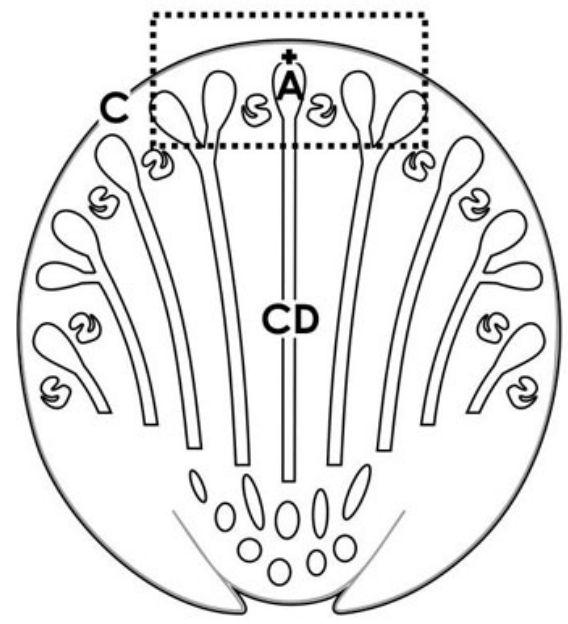

Figure 1. Schematic illustration shows section planes to obtain comparable views to the renal stem/progenitor cell niche. (a) First the kidney is cut between the upper and lower pole. (b) On the resulting section collecting duct (CD) tubules line from the papilla to the capsule (C). In the outer cortex a CD tubule forms an ureteric bud derived ampulla (A) enclosing epithelial stem/progenitor cells. The basal lamina at the tip of a CD ampulla is marked by a cross $(+)$.

\section{Embedding of specimens in resin}

Before embedding tissue was washed with $0.15 \mathrm{M}$ sodium cacodylate buffer and dehydrated in graded series of ethanols. Finally the specimens were embedded in Epon (Fluka), which was polymerized at $60^{\circ} \mathrm{C}$ for $48 \mathrm{~h}$.

\section{Transmission electron microscopy}

Semithin and ultrathin sections were performed with a diamond knife on an ultramicrotome EM UC6 (Leica GmbH, Wetzlar, Germany). Semithin sections were placed on glass slides and stained by Richardson solution. Ultrathin sections were collected onto slot grids coated with 1.5\% Pioloform (Plano, Wetzlar, Germany) and contrasted using $2 \%$ uranyl acetate and lead citrate as earlier described [37]. Specimens were finally examined at $80 \mathrm{kV}$ using an EM 902 transmission electron microscope (Zeiss, Oberkochen, Germany). Images were taken with a digital camera and thereafter processed with Corel DRAW Graphic Suite X5 (Corel Corporation).

\section{Amount of analyzed specimens}

A total of 37 exactly orientated renal stem cell niches was analyzed for the present study. All of the specimens were analyzed at least in triplicates. Performed experiments are in accordance with the Animal Ethics Committee, University of Regensburg, Regensburg, Germany.

Definition of structures within the renal stem/progenitor cell niche

In the present paper the embryonic part of neonatal kidney was analyzed. In consequence, the nomenclature of previously published papers was used [22].

\section{Results}

Vertical view to the renal stem/progenitor cell niche During nephron induction morphogenetic molecules are reciprocally exchanged between the tip of an ureteric bud derived CD ampulla and surrounding mesenchymal stem/ progenitor cells. Thus, the interface between epithelial cells enclosed in the CD ampulla and mesenchymal stem/ progenitor cells is of outmost relevance for the transmission of morphogenetic signals. To analyze in detail morphological aspects of this important site, a block of renal parenchyma has to be orientated (Flgure 1a). Consequently, sections were always made in parallel to the lumen of lining collecting duct tubules. Following this perspective, at their cortical endings a CD ampulla, mesenchymal stem/progenitor cells and the covering organ capsule are found as a constant histological ensemble. Applying this orientation an always comparable view in histological sections is obtained (Figure 1b). For clear identification the basal lamina at the tip of a CD ampulla is marked by a cross on each micrograph.

\section{Optical microscopy}

A renal stem/progenitor cell niche is demonstrated on a semithin section made from the outer cortical parenchyma of specimens fixed by conventional glutaraldehyde (Figure 2a). The tip of a $\mathrm{CD}$ ampulla enclosing epithelial stem/progenitor cells is seen in a distance between 16 and $20 \mu \mathrm{m}$ beyond the organ capsule. Between both two layers of mesenchymal stem/progenitor cells are found. Even this low magnification shows that an interstitial interface separates epithelial and mesenchymal stem/progenitor cells.

\section{Analysis by transmission electron microscopy}

To visualize better the interface between epithelial and mesenchymal cells within the renal stem/progenitor cell niche, specimens were analyzed by transmission electron microcopy (Figures 2b, 2c and 3-5). 

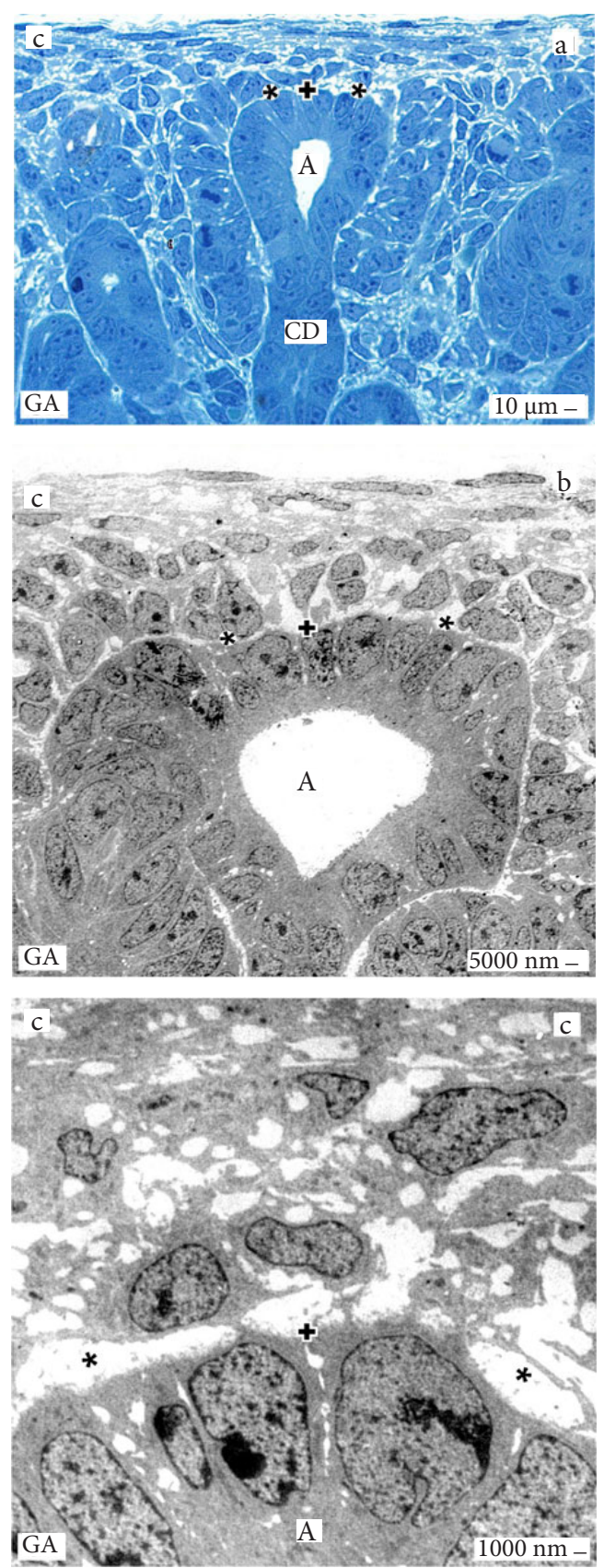

Figure 2. View to the stem/progenitor cell niche after fixation by conventional glutaraldehyde (GA) solution. (a) A semithin section is made along the axis of a collecting duct (CD) tubule. In the outer cortex an ampulla (A) with enclosed epithelial stem/progenitor cells is seen. Between the capsule (C) and the ampulla two layers of mesenchymal cells are present. Both types of stem/progenitor cells are separated by a striking interface (asterisk). (b) Transmission electron microscopy depicts that the interface (asterisk) separates epithelial from mesenchymal stem/progenitor cells. (c) Higher magnification shows projections of mesenchymal cells traversing the interface to contact the basal aspect of a CD ampulla. The basal lamina at the tip of a CD ampulla is marked by a cross (+).

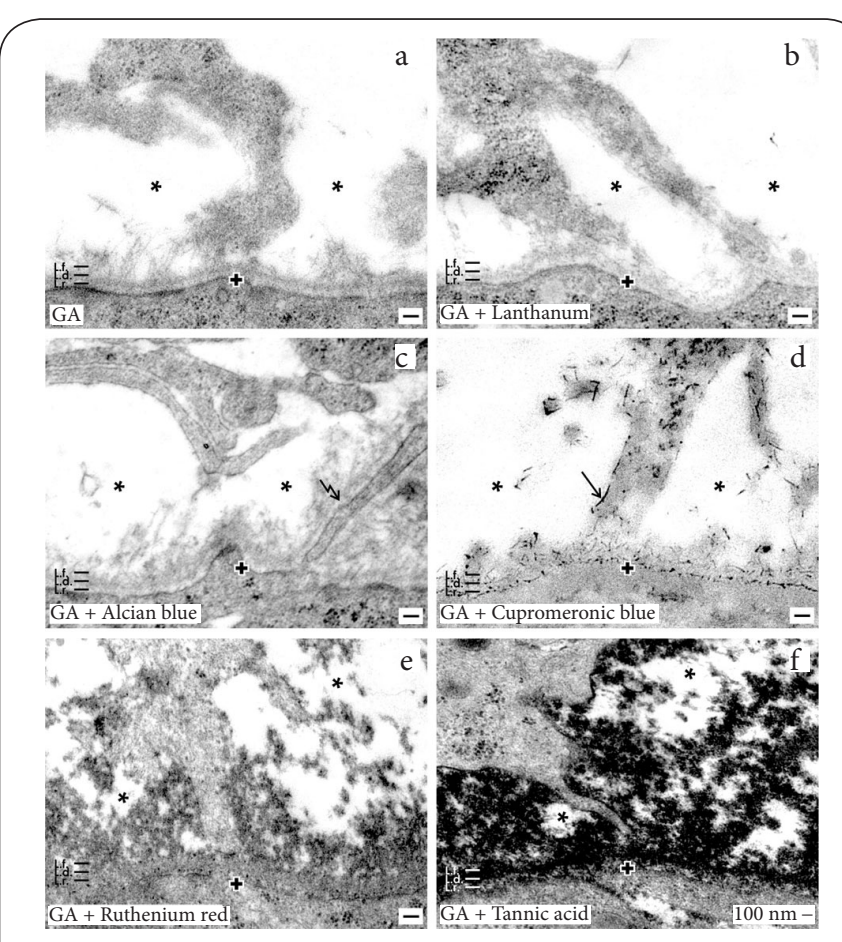

Figure 3. Analysis of the interface within the renal stem/ progenitor cell niche by transmission electron microscopy. Specimens were fixed by glutaraldehyde (a), glutaraldehyde including lanthanum (b), alcian blue (c), cupromeronic blue (d), ruthenium red (e) or tannic acid (f). It is demonstrated that mesenchymal and epithelial cells are separated by an interface (asterisk). Projections of mesenchymal cells cross the interface to contact a CD ampulla. (a) Fixation by glutaraldehyde exhibits a bright interface separating mesenchymal and epithelial cells. (b) Fixation by glutaraldehyde including lanthanum shows an inconspicuous interface. (c) Fixation by glutaraldehyde including alcian blue illustrates that projections of mesenchymal cells are enveloped by filigree extracellular matrix in form of a sleeve (serrated arrow). (d) Fixation by glutaraldehyde including cupromeronic blue illustrates buckles of proteoglycans on the basal aspect of epithelial stem/progenitor cells and on mesenchymal cell projections (arrow). (e) Fixation by ruthenium red shows that a labeled band of extracellular matrix surrounds a CD ampulla. In some areas the interface is bright, while in other areas a punctuate pattern of extracellular matrix is seen. (f) Fixation by glutaraldehyde containing tannic acid shows a dense coat on the basal lamina of a CD ampulla. Distinct areas of the interface are bright, while others contain filigree extracellular matrix. The basal lamina (lamina rara (L.r.), lamina densa (L.d.) and lamina fibroreticularis (L.f.)) at the tip of a CD ampulla is marked by a cross $(+)$.

\section{Fixation by glutaraldehyde}

For control, in the first series of experiments specimens were fixed by conventional glutaraldehyde solution (Figures 2, 3a and 4a). Between the inner side of the organ capsule and the tip of a $\mathrm{CD}$ ampulla two layers of mesenchymal cells become visible (Figure 2b). It can be further seen that at the basal aspect of a 


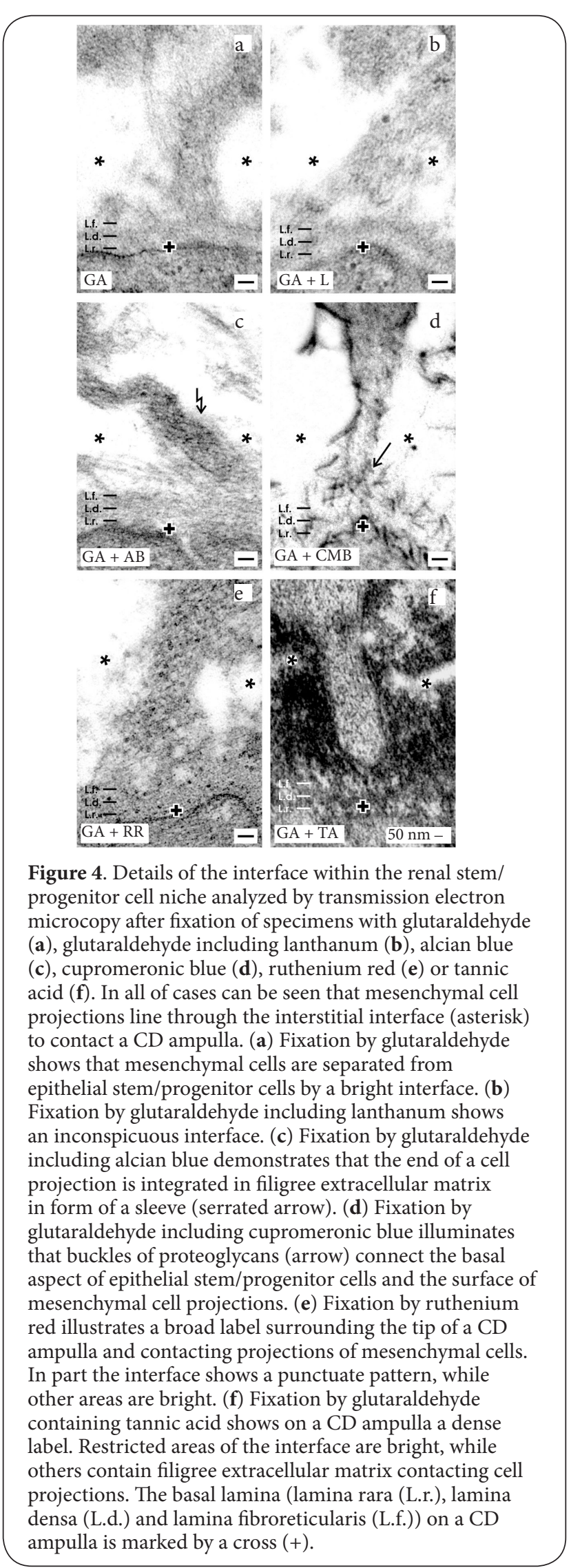

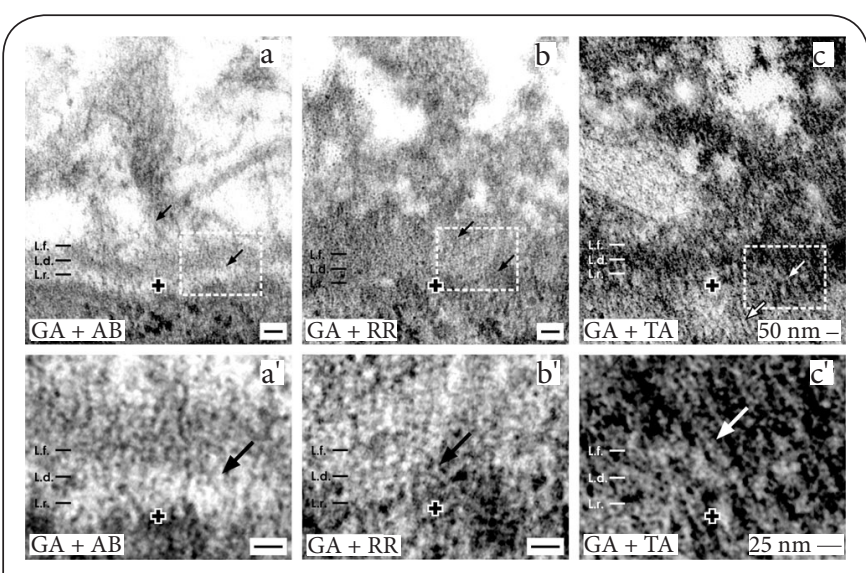

Figure 5. Contact site between mesenchymal cell projections and epithelial cells within the renal stem/progenitor cell niche in transmission electron microscopy. Specimens were fixed in glutaraldehyde containing either alcian blue $\left(\mathbf{a}, \mathbf{a}^{\mathbf{a}}\right)$, ruthenium red $\left(\mathbf{b}, \mathbf{b}^{\prime}\right)$ or tannic acid $\left(\mathbf{c}, \mathbf{c}^{\prime}\right)$. It can be seen that endings of mesenchymal cell projections are specifically mounted at a CD ampulla. Tunneling nanotubes (arrows) in the interior of cell projections line through the basal lamina of a CD ampulla to contact epithelial cells. The basal lamina (lamina rara (L.r.), lamina densa (L.d.) and lamina fibroreticularis (L.f.)) on a CD ampulla is marked by a cross $(+)$.

CD ampulla the epithelial and mesenchymal stem/progenitor cells do not stand in close contact but are separated by a bright interface. The next higher magnification illuminates that some projections of mesenchymal stem/progenitor cells are crossing the interface to contact the basal lamina of a CD ampulla (Figure 2c).

An increased magnification depicts that a consistently developed basal lamina covers epithelial stem/progenitor cells at the tip of a CD ampulla (Figure 3a). The basal lamina consists of a clearly visible lamina rara (L.r.), a lamina densa (L.d.) and a lamina fibroreticularis (L.f.). Only few microfibers protrude from the lamina fibroreticularis towards the interface. Between mesenchymal stem/progenitor cells and the basal aspect of a CD ampulla a remarkably wide but fully inconspicuously looking interface is registered. Projections from mesenchymal stem/progenitor cells line occasionally through the interface to contact the basal lamina of a CD ampulla. This spatial arrangement reflects a special contact via distance between mesenchymal and epithelial stem/progenitor cells. All of the morphological details appear to be well preserved, when specimens are fixed by conventional GA fixation (Figures $\mathbf{2} \mathbf{b}, \mathbf{2 c}$ ). In so far the micrographs seem to reflect the natural situation. Due to these reasons the interface between both types of renal stem/progenitor cells cannot be ascribed to an artifact.

Although the interface is bright, it seems to be probable that the related space is not fully filled by liquid but is kept wide by molecules, which cannot be contrasted by conventional techniques. To unveil hidden structural details, alternative fixation and contrasting techniques were applied. 
Minuth et al. Stem Cell Biology and Research 2014,

\section{Fixation by glutaraldehyde containing lanthanum}

In the second series of experiments GA solution containing lanthanum was used for fixation (Figures $\mathbf{3 b}$ and $\mathbf{4 b}$ ). High (Figure $\mathbf{3 b}$ ) and very high (Figure $\mathbf{4 b}$ ) magnifications of the renal stem/ progenitor cell niche are focussing to the basal side of epithelial stem/progenitor cells within the tip of a CD ampulla, the mesenchymal stem/progenitor cells and their projections. Although not so well contrasted, it can be recognized that a consistently developed basal lamina covers the tip of a CD ampulla. As in the previous series a bright but unconspicuously looking interstitial interface is observed. The projections of mesenchymal stem/progenitor cells line through the interface. In many cases their endings are connected with microfibers deriving from the lamina fibroreticularis. Further differences between specimens fixed by GA (Figures $\mathbf{2} \mathbf{b}, \mathbf{2} \mathbf{c}$ and $\mathbf{3 a}, \mathbf{4} \mathbf{a}$ ) and GA containing lanthanum (Figures $\mathbf{3 b}$ and $\mathbf{4 b}$ ) are not registered.

\section{Fixation by glutaraldehyde containing alcian blue} Specimens fixed by glutaraldehyde containing alcian blue depict that a three-layered basal lamina covers the basal aspect of a CD ampulla (Figures $\mathbf{3 c}, \mathbf{4 c}$ and $\mathbf{5 a}, \mathrm{a}^{\mathbf{}}$ ). Mesenchymal cells are separated from epithelial stem/progenitor cells also in this case by a bright interstitial interface. Cell projections of mesenchymal stem/progenitor cells line through the interface and contact the basal lamina of epithelial cells. Label for alcian blue further illustrates that the plasma membrane of mesenchymal cell projections is sharply contoured. Following individual projections to their endings, it can be seen that tiny fibers of extracellular matrix embrace them like a sleeve. It is illustrated that the end of cell projections is stabilized on the basal lamina of a CD ampulla by microfibers. It can be further recognized that tunneling nanotubes within the interior of cell projections line through the basal lamina of a CD ampulla to contact epithelial cells (Figure 5a). This important result demonstrates that mesenchymal cell projections do not dangle in the interface but are structurally and functionally connected.

Fixation by glutaraldehyde containing cupromeronic blue Specimens fixed by glutaraldehyde containing cupromeronic blue also demonstrate that epithelial and mesenchymal stem/ progenitor cells are separated by a wide interface (Figures $\mathbf{3 d}$ and 4d). Most interestingly, applying cupromeronic blue contrasting buckles of proteoglycans are seen at the basal aspect of a CD ampulla. In particular the basal plasma membrane of epithelial stem/progenitor cells and the lamina fibroreticularis of the basal lamina show labeled proteoglycans. Also the surface of mesenchymal cell projections is covered by numerous buckles of proteoglycans. Further can be seen that the endings of mesenchymal cell projections contact the basal lamina of a CD ampulla. At this contact site proteoglycans fuse with each other forming a sleeve. Analyzing this construction it conveys the impression that the contact between mesenchymal cell projection and the basal lamina of a CD ampulla is specially adapted.

Fixation by glutaraldehyde containing ruthenium red Specimens fixed by glutaraldehyde containing ruthenium red reveal that the basal lamina of a CD ampulla appears to be completely different as compared to previous series (Figures $3 e$, $\mathbf{4 e}$ and $\mathbf{5 b}, \mathbf{b}^{\prime}$ ). Applying ruthenium red contrasting the typical three-laminar structure at basal lamina of a CD ampulla is not visible anymore. Instead a single broad band of intense ruthenium red label surrounds the CD ampulla. In some parts the interstitial interface is bright, while other parts are filled by clouds of a punctuate but fligree extracellular matrix. Further can be recognized that projections of mesenchymal cells line through the interstitial interface to contact the basal lamina on a CD ampulla. Often it is seen that filigree extracellular matrix surrounds the endings of projections in form of a basket. Very high magnification depicts that tunneling nanotubes line from the endings of mesenchymal cell projections through the basal lamina of a CD ampulla to contact epithelial cells (Figure 5b). This results speaks in favor that the contact site is not accidental but long-lasting and functional.

\section{Fixation by glutaraldehyde containing tannic acid}

Specimens fixed by glutaraldehyde containing tannic acid show that the complete basal lamina at the tip of a CD ampulla is covered by an electron-dense coat (Figures $\mathbf{3 f}, \mathbf{4 f}$ and $\mathbf{5 c}, \mathbf{c}^{\prime}$ ). However, the band measures only half of tickness detected in the series with ruthenium red label (Figures $\mathbf{3 e}, \mathbf{4 e}$ and $\left.\mathbf{5} \mathbf{b}, \mathbf{b}^{\prime}\right)$. Contrasting by tannic acid further depicts that the lamina rara is discontinuously contrasted, while the lamina densa and lamina fibroreticularis reflect a continuous labeling profile. Restricted areas of the interface are bright, while in other areas tannic acid label unveils to a high degree clouds of filigree extracellular matrix. Further the surface of mesenchymal cell projections is sharply contrasted. Also in this series can be seen that projections of mesenchymal cells cross the interface to contact via tunneling nanotubes epithelial cells within a CD ampulla (Figure $\mathbf{5 c}$, $\mathbf{c}^{\prime}$ ). In most of cases it is observed that cell projections and especially their endings are covered by a fine-granular texture of extracellular matrix forming a sleeve. Also this result point out that the contact site is functionally adapted.

\section{Site-specific label within the niche}

Fixation of specimens by GA containing tannic acid demonstrates most forcefully that at the tip of a CD ampulla intense label is found at the basal lamina, within distinct areas of the adjacent interstitial interface and on the surface of neighboring mesenchymal stem/progenitor cells (Figures $\mathbf{3 f}, \mathbf{4} \mathbf{f}$ and $\mathbf{5} \mathbf{c}, \mathbf{c}^{\prime}$ ). At the lateral side of a $C D$ ampulla intensity of tannic acid label decreases, while at the ampulla neck label is lacking. Since label for tannic acid is also missing in neighboring Comma-, $\mathrm{S}$-shaped bodies and further matured nephrons, there is a strong evidence that a specific structural environment within 
the renal stem/progenitor cell niche is detected. Further on, not the total volume of the interface but only distinct areas exhibiting filigree extracellular matrix is recognized by tannic acid. All these results speak for a specific contrasting and not for a unspecific background signal.

\section{Discussion and conclusion}

During development of the kidney the stem/progenitor cell niche is found in the outer cortex of parenchyma and in an always close neighborhood to the inner side of the organ capsule [25]. At this special site formation of a nephron starts [38]. This process pushes epithelial cells within the tip of a CD ampulla in a correct position to surrounding nephrogenic mesenchymal stem/progenitor cells. Then a reciprocal exchange of morphogenetic factors is leading to an aggregation of the nephron anlage [30-33]. While the molecular-biological action of involved morphogenetic factors was extensively investigated, related literature does not inform about the mechanism of molecule transmission. Generally, it is anticipated that a close spatial contact between interacting stem/progenitor cells exists so that only a short route for diffusion of molecules must be overcome. Under such ideal conditions dilution of secreted molecules appears to be minimized and fast passing of the diffusion route seems to be most probable [39].

However, actual data exhibits that these assumptions are not in agreement with the morphological situation found within the renal stem/progenitor cell niche. As well a look through the optical microscope (Figure 2a) as analysis in transmission electron microscopy (Figures $\mathbf{2 b}, \mathbf{2 c}$ and $\mathbf{3}-\mathbf{5}$ ) informs that both types of stem/progenitor cells do not stand as expected in close contact but are spatially separated by a wide interface.

\section{Diffusion of morphogenetic signals via the interface} When one thinks on diffusion theory, epithelial stem/pro-genitor cells enclosed within the tip of a CD ampulla secrete morphogenetic factors at the basolateral plasma membrane (Figure 6a). Further assuming that the interstitium is filled by liquid, each of secreted molecules such as FGFs, Wnt9b or LIF must diffuse first through three layers of the basal lamina at a $C D$ ampulla tip and then through the wide interface, before it can contact receptors on the plasma membrane of mesenchymal cells. Considering a reciprocal exchange, for example GDNF secreted by mesenchymal cells must take the vice versa route for binding on receptors at the basolateral plasma membrane of epithelial stem/progenitor cells [32].

Arguments on the basis of earlier findings in transmission electron microscopy speak for an unhindered shuttling of morphogenetic molecules between epithelial and mesenchymal stem/progenitor cells by diffusion. When those specimens were fixed by conventional glutaraldehyde solution, the interstitial interface within the renal stem/progenitor cell niche is always seen as a wide but fully inconspicuously looking space (Figures $\mathbf{2 b}, \mathbf{2 c}$ and $\mathbf{3 a}, \mathbf{4 a}$ ). This kind of fixation
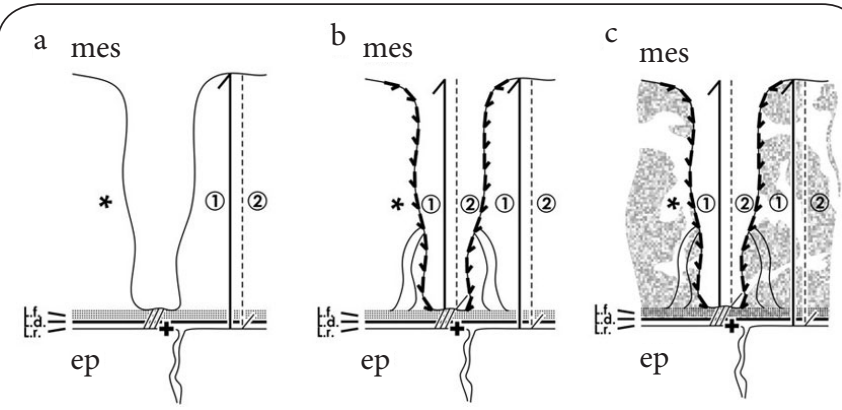

Figure 6. Schematic illustration shows possible routes for transmission of morphogenetic factors between mesenchymal (mes) and epithelial (ep) cells via the interface within the renal stem/progenitor cell niche. (a) Fixation of specimens by GA suggests that morphogenetic factors are exchanged between epithelial (1) and mesenchymal (2) cells by diffusion via the bright interface. (b) Fixation of specimens by GA containing lanthanum, alcian blue and cupromerionic blue suggests that projections of mesenchymal cells contact the basal aspect of epithelial cells supporting an exchange via cell to cell contacts and diffusion. (c) Fixation of specimens by GA containing ruthenium red and tannic acid elucidates that projections of mesenchymal cells are integrated in filigree extracellular matrix. In this case morphogenetic molecules can be exchanged by diffusion, mesenchymal cell projections and modulating extracellular matrix. The basal lamina consisting of a lamina rara (L.r.), lamina densa (L.d.) and lamina fibroreticularis (L.f.) at the tip of a CD ampulla is marked by a cross $(+)$.

suggests that the interface is reflecting an extended space solely filled with interstitial fluid. It is obvious that such a result also implies unlimited diffusion of secreted molecules as it was frequently investigated by transgenic mice and isolated metanephric mesenchyme in culture $[\mathbf{4 0 , 4 1 ]}$.

\section{Morphogenetic signaling via cell to cell contacts}

Experimental approaches analyzing transmission of morphogenetic signals between metanephric mesenchyme and inducer tissue was earlier performed by transfilter culture experiments [42]. In those experiments mesenchyme of embryonic kidney was isolated and placed on one side of a nitrocellulose filter. To induce tubule formation, spinal cord was fixed on the other side by agar coating. Replacing nitrocellulose against a Nuclepore ${ }^{\circledR}$ filter it was further demonstrated that not the diffusion of morphogenetic molecules but a transfilter contact between projections of interacting cells is necessary to induce tubule formation [43]. In this coherence it was also described that within one hour cytoplasmic processes emerge through an interposed filter. Then further 16 to 24 hours of direct cell to cell contacts via projections are needed for completion of tubule induction. Although a diffusion of morphogenetic molecules cannot be excluded, performed transfilter experiments demonstrate that cell to cell contacts between interacting tissues are essential for tubule induction. It is generally accepted that transfer of cellular material can take place via tunneling nanotubes [44]. As well recently 
Minuth et al. Stem Cell Biology and Research 2014,

http://www.hoajonline.com/journals/pdf/2054-717X-1-4.pdf

doi: 10.7243/2054-717X-1-4

published literature as here presented data illustrates that projections of mesenchymal cells line through the interface, penetrate the basal lamina at a CD ampulla tip and contact via tunneling nanotubes the basal aspect of epithelial stem/ progenitor cells (Figures 3-5) [24]. Regarding these actual results, it has to be considered that morphogenetic signaling principally can take place by both diffusion via the interstitial interface and by cell to cell contacts (Figure $6 \mathbf{b}$ ).

\section{Transmission of signals via modulating extracellular matrix}

Literature further informs that beside morphogenetic molecules also extracellular matrix especially proteoglycans are involved in the process of nephron formation [21,45-48]. The resulting question is, whether extracellular matrix detected by improved fixation of specimens within the interface of the renal stem/progenitor cell niche indicates an association with transmission of these morphogenetic signals (Figure $6 \mathrm{c}$ ).

Fixation of specimens by GA containing alcian blue (Figures $3 c$ $\mathbf{4 c}$ and $\mathbf{5 a}, \mathrm{a}^{\prime}$ ) illustrates that numerous microfibers enclose the endings of mesenchymal stem/progenitor cell projections. Contrasting by cupromeronic blue further shows that the surface of cell projections is covered by numerous buckles of proteoglycans (Figures $\mathbf{3 d}$ and $\mathbf{4 d}$ ). At the endings of mesenchymal cell projections and the basal lamina of a CD ampulla proteoglycans form a zone in form of a connecting cylinder. Further on, alternative fixation of specimens by ruthenium red (Figures $\mathbf{3 e}, \mathbf{4 e}$ and $\mathbf{5 b}, \mathbf{b}^{\prime}$ ) and tannic acid (Figures $\mathbf{3 f}, \mathbf{4} \mathbf{f}$ and $\mathbf{5 c}, \mathbf{c}^{\prime}$ ) depicts that cell projections are specifically embraced by a punctuate pattern of extracellular matrix. In other words, the illustrated cell projections are not dangling in the inter-face but are structurally featured by surrounding filigree extra cellular matrix.

In additon, in all of the cases can be seen that the contact of mesenchymal cell projections and extracellular matrix is not accidental but is sophisticatedly designed. Comparing further the different kinds of fixation protocols, it is obvious that beside mesenchymal cell projections different textures of extracellular matrix in form of microfibers, buckles and granules are contained in the interface [49]. Regarding diversity and special microarchitecture of detected matrix in combination with individually integrated mesenchymal cell projections and special contacts via tunneling nanotubes on the basal aspect of epithelial stem/progenitor cells, all of that appears as a structural ensemble. It further suggests that illustrated extracellular matrix within the interstitial interface acts as a zone controlling transmission of morphogenetic information. Thus, the present investigation demonstrates that the interstitial interface within the renal stem/progenitor cell niche is not a space simply filled by liquid. Instead, it contains an unexpected large amount of filigree extracellular matrix. Further projections of mesenchymal cells do not incidentally line through it, but are specifically integrated in illustrated matrix. Although these customized morphological characteristics do not yet inform about physiological properties, they declare an earlier not considered spatial separation of epithelial and mesenchymal cells within the renal stem/progenitor cell niche. To what extent illustrated extracellular matrix is a basic structural element or whether it is able to modulate the transmisson of morphogenetic molecules has to be investigated.

\section{Future perspectives}

Fixation of specimens by conventional glutaraldehyde solution exhibits in transmission electron microscopy that epithelial and mesenchymal cells within the renal stem/progenitor cell niche are separated by a wide but inconspicuously looking interface. In contrast, improved fixation of specimens by glutaraldehyde containing alcian blue, cupromeronic blue, ruthenium red or tannic acid make visible that the interface is filled by different textures of filigree extracellular matrix. In between projections of mesenchymal cells traverse the interface to contact epithelial cells via tunneling nanotubes. This special microarchitecture explains on the one hand the constant distance between epithelial and mesenchymal cells. On the other hand the results raise the question, whether reciprocal exchange of morphogenetic factors solely occurs via diffusion or if also illustrated extracellular matrix and cell projections are involved. Further exploration of newly detected microenvironment within the renal stem/progenitor cell niche will help to learn from nature and to give new impact for regeneration of diseased renal parenchyma by drugs featuring controlled development.

\section{Competing interests}

The authors declare that they have no competing interests.

Authors' contributions

\begin{tabular}{|l|c|c|}
\hline Authors' contributions & WWM & LD \\
\hline Research concept and design & $\checkmark$ & -- \\
\hline Collection and/or assembly of data & -- & $\checkmark$ \\
\hline Data analysis and interpretation & $\checkmark$ & $\checkmark$ \\
\hline Writing the article & $\checkmark$ & -- \\
\hline Critical revision of the article & -- & $\checkmark$ \\
\hline Final approval of article & $\checkmark$ & -- \\
\hline Statistical analysis & -- & -- \\
\hline
\end{tabular}

\section{Acknowledgement}

The authors thank the Institute of Molecular and Cellular Anatomy, University of Regensburg for financial support and technical assistance.

\section{Publication history}

Editor: Toru Hosoda, Tokai University, Japan.

EIC: Prasad S Koka, Haffkine Institute for Training, Research \& Testing, India.

Received: 16-Sep-2014 Final Revised: 19-Nov-2014

Accepted: 10-Dec-2014 Published: 17-Dec-2014

\section{References}

1. Caddeo G, Williams ST, Mclntyre CW and Selby NM. Acute kidney injury in urology patients: incidence, causes and outcomes. Nephrourol Mon. 2013; 5:955-61. | Article | PubMed Abstract | PubMed Full Text 
2. Kalamas $A G$ and Niemann CU. Patients with chronic kidney disease. Med Clin North Am. 2013; 97:1109-22. I Article I PubMed

3. Bayliss $\mathrm{G}$. Initiation of dialysis: a mini-review of a changing paradigm. Cardiovasc Hematol Disord Drug Targets. 2014; 14:165-9. | Article | PubMed

4. Tong A, Chapman JR, Wong G and Craig JC. Perspectives of transplant physicians and surgeons on reimbursement, compensation, and incentives for living kidney donors. Am J Kidney Dis. 2014; 64:622-32. | Article I PubMed

5. Aggarwal S, Moggio A and Bussolati B. Concise review: stem/progenitor cells for renal tissue repair: current knowledge and perspectives. Stem Cells Trans/ Med. 2013; 2:1011-9. | Article I PubMed Abstract | PubMed Full Text

6. Herrera M and Mirotsou M. Stem cells: potential and challenges for kidney repair. Am J Physiol Renal Physiol. 2014; 306:F12-23. | Article | PubMed

7. de Almeida DC, Donizetti-Oliveira C, Barbosa-Costa P, Origassa CS and Camara NO. In search of mechanisms associated with mesenchymal stem cell-based therapies for acute kidney injury. Clin Biochem Rev. 2013; 34:131-44. | PubMed Abstract | PubMed Full Text

8. Winyard PJ and Price KL. Experimental renal progenitor cells: repairing and recreating kidneys? Pediatr Nephrol. 2014; 29:665-72. | Article | PubMed

9. Wang $Y, H e J$, Pei $X$ and Zhao W. Systematic review and meta-analysis of mesenchymal stem/stromal cells therapy for impaired renal function in small animal models. Nephrology (Carlton). 2013; 18:201-8. | Article | PubMed

10. Burst V, Putsch F, Kubacki T, Volker LA, Bartram MP, Muller RU, Gillis M, Kurschat CE, Grundmann F, Muller-Ehmsen J, Benzing T and Teschner S. Survival and distribution of injected haematopoietic stem cells in acute kidney injury. Nephrol Dial Transplant. 2013; 28:1131-9. | Article I PubMed

11. Little MH. Renal organogenesis: what can it tell us about renal repair and regeneration? Organogenesis. 2011; 7:229-41. | Article | PubMed Abstract | PubMed Full Text

12. Little MH and McMahon AP. Mammalian kidney development: principles, progress, and projections. Cold Spring Harb Perspect Biol. 2012; 4. | Article | PubMed Abstract | PubMed Full Text

13. Togel FE and Westenfelder C. Kidney protection and regeneration following acute injury: progress through stem cell therapy. Am J Kidney Dis. 2012; 60:1012-22. | Article | PubMed

14. Ebrahimi B, Eirin A, Li Z, Zhu XY, Zhang X, Lerman A, Textor SC and Lerman LO. Mesenchymal stem cells improve medullary inflammation and fibrosis after revascularization of swine atherosclerotic renal artery stenosis. PLoS One. 2013; 8:e67474. | Article | PubMed Abstract | PubMed Full Text

15. Zhu XY, Lerman A and Lerman LO. Concise review: mesenchymal stem cell treatment for ischemic kidney disease. Stem Cells. 2013; 31:1731-6. | PubMed Abstract | PubMed Full Text

16. Minuth WW, Denk L. Search for chemically defined culture medium to assist initial regeneration of diseased renal parenchyma after stem/ progenitor cell implantation. Int J Stem Cell ResTransplant. 2013; 1:202. | Article

17. Nigam SK. Concise review: can the intrinsic power of branching morphogenesis be used for engineering epithelial tissues and organs? Stem Cells Transl Med. 2013; 2:993-1000. | Article | PubMed Abstract | PubMed Full Text

18. Faa G, Gerosa C, Fanni D, Monga G, Zaffanello M, Van Eyken P and Fanos V. Morphogenesis and molecular mechanisms involved in human kidney development. J Cell Physiol. 2012; 227:1257-68. | Article | PubMed

19. Piludu M, Fanos V, Congiu T, Piras M, Gerosa C, Mocci C, Fanni D, Nemolato S, Muntoni S, lacovidou N and Faa G. The pine-cone body: an intermediate structure between the cap mesenchyme and the renal vesicle in the developing nod mouse kidney revealed by an ultrastructural study. J Matern Fetal Neonatal Med. 2012; 25:72-5. I

\section{Article I PubMed}

20. Meyer TN, Schwesinger C, Bush KT, Stuart RO, Rose DW and Shah MM. Spatiotemporal regulation of morphogenetic molecules during in vitro branching of the isolated ureteric bud: toward a model of branching through budding in the developing kidney. Dev Biology. 2004; 275:4467. | Article

21. Nigam SK and Bush KT. Growth factor-heparan sulfate "switches" regulating stages of branching morphogenesis. Pediatr Nephrol. 2014; 29:727-35. | Article | PubMed

22. Minuth WW, Denk L, Miess C and Glashauser A. Peculiarities of the extracellular matrix in the interstitium of the renal stem/progenitor cell niche. Histochem Cell Biol. 2011; 136:321-34. I Article I PubMed

23. Bush KT, Martovetsky G and Nigam SK. Relevance of ureteric bud development and branching to tissue engineering, regeneration and repair in acute and chronic kidney disease. Curr Opin Organ Transplant. 2014; 19:153-61. | Article | PubMed

24. Minuth WW and Denk L. Cell projections and extracellular matrix cross the interstitial interface within the renal stem/progenitor cell niche: accidental, structural or functional cues? Nephron Exp Nephrol. 2012; 122:131-40. | Article | PubMed

25. Minuth $W$ and Denk L. Structural links between the renal stem/ progenitor cell niche and the organ capsule. Histochem Cell Biol. 2014; 141:459-471. I Pdf

26. Michos O. Kidney development: from ureteric bud formation to branching morphogenesis. Curr Opin Genet Dev. 2009; 19:484-90. I Article | PubMed Abstract | PubMed Full Text

27. Dressler GR. Advances in early kidney specification, development and patterning. Development. 2009; 136:3863-74. I Article I PubMed Abstract | PubMed Full Text

28. Sariola H. Nephron induction revisited: from caps to condensates. Curr Opin Nephrol Hypertens. 2002; 11:17-21. I Article I PubMed

29. Nishinakamura R. Stem cells in the embryonic kidney. Kidney Int. 2008; 73:913-7. | Article | Pubmed

30. Davies JA. Morphogenesis of the metanephric kidney. ScientificWorldJournal. 2002; 2:1937-50. I Article I PubMed

31. Oxburgh L, Brown AC, Fetting J and Hill B. BMP signaling in the nephron progenitor niche. Pediatr Nephrol. 2011; 26:1491-7. | Article | PubMed Abstract | PubMed Full Text

32. Chai OH, Song CH, Park SK, Kim W and Cho ES. Molecular regulation of kidney development. Anat Cell Biol. 2013; 46:19-31. I Article I PubMed Abstract | PubMed Full Text

33. Carroll TJ and Das A. Defining the signals that constitute the nephron progenitor niche. J Am Soc Nephrol. 2013; 24:873-6. | Article I PubMed

34. Minuth WW and Denk L. Illustration of extensive extracellular matrix at the epithelial-mesenchymal interface within the renal stem/progenitor cell niche. BMC Clin Pathol. 2012; 12:16. I Article

35. Hasko JA and Richardson GP. The ultrastructural organization and properties of the mouse tectorial membrane matrix. Hear Res. 1988; 35:21-38. | Article | PubMed

36. Rothenburger M, Volker W, Vischer P, Glasmacher B, Scheld HH and Deiwick M. Ultrastructure of proteoglycans in tissue-engineered cardiovascular structures. Tissue Eng. 2002; 8:1049-56. | Article | PubMed

37. Minuth WW, Denk L, Meese C, Rachel R and Roessger A. Ultrastructural insights in the interface between generated renal tubules and a polyester interstitium. Langmuir. 2009; 25:4621-7. | Article I PubMed

38. Nigam SK and Shah MM. How does the ureteric bud branch? J Am Soc Nephrol. 2009; 20:1465-9. I Article I PubMed

39. Lander AD. Morpheus unbound: reimagining the morphogen gradient. Cell. 2007; 128:245-56. | Article | PubMed

40. Halt $\mathrm{K}$ and Vainio S. Coordination of kidney organogenesis by Wnt signaling. Pediatr Nephrol. 2014; 29:737-44. I Article I PubMed Abstract | PubMed Full Text

41. Matsumoto S, Fujii S, Sato A, Ibuka S, Kagawa Y, Ishii M and Kikuchi A. A combination of Wnt and growth factor signaling induces Arl4c 
Minuth et al. Stem Cell Biology and Research 2014,

http://www.hoajonline.com/journals/pdf/2054-717X-1-4.pdf

expression to form epithelial tubular structures. EMBO J. 2014; 33:70218. | Article | PubMed

42. Grobstein $C$. Trans-filter induction of tubules in mouse metanephrogenic mesenchyme. Exp Cell Res. 1956; 10:424-40. | Article | PubMed

43. Saxen $L$ and Lehtonen $E$. Transfilter induction of kidney tubules as a function of the extend and duration of intercellular contacts. J Embryol Exp Morphol. 1978; 47:97-109. | Pdf

44. Domhan S, Ma L, Tai A, Anaya Z, Beheshti A, Zeier M, Hlatky L and Abdollahi A. Intercellular communication by exchange of cytoplasmic material via tunneling nano-tube like structures in primary human renal epithelial cells. PLoS One. 2011; 6:e21283. | Article | PubMed Abstract | PubMed Full Text

45. Mathew S, Chen X, Pozzi A and Zent R. Integrins in renal development. Pediatr Nephrol. 2012; 27:891-900. | Article | PubMed

46. Das A, Tanigawa S, Karner CM, Xin M, Lum L, Chen C, Olson EN, Perantoni $\mathrm{AO}$ and Carroll TJ. Stromal-epithelial crosstalk regulates kidney progenitor cell differentiation. Nat Cell Biol. 2013; 15:1035-44. | Article | PubMed Abstract | PubMed Full Text

47. Paroly SS, Wang F, Spraggon L, Merregaert J, Batourina E, Tycko B, Schmidt-Ott KM, Grimmond S, Little M and Mendelsohn C. Stromal protein Ecm 1 regulates ureteric bud patterning and branching. PLOS One. 2013; 8:e84155. | Article | PubMed Abstract | PubMed Full Text

48. Fetting JL, Guay JA, Karolak MJ, lozzo RV, Adams DC, Maridas DE, Brown $A C$ and Oxburgh L. FOXD1 promotes nephron progenitor differentiation by repressing decorin in the embryonic kidney. Development. 2014; 141:17-27. | Article | PubMed

49. Minuth WW and Denk L. The Interstitial Interface within the Renal Stem/Progenitor Cell Niche Exhibits an Unique Microheterogeneous Composition. Int J Mol Sci. 2013; 14:13657-69. | Article | PubMed Abstract | PubMed Full Text

\section{Citation:}

Minuth WW and Denk L. Separating and connecting properties of the interface within the renal stem/progenitor cell niche. Stem Cell Biol Res. 2014; 1:4. http://dx.doi.org/10.7243/2054-717X-1-4 